\title{
EFICÁCIA DE HERBICIDAS NO CONTROLE EM PRÉ-EMERGÊNCIA DE PLANTAS DANINHAS NA CULTURA DO ARROZ
}

\author{
Fernando Tadeu de Carvalho ${ }^{1}$, Maurício Alessandro Cavazzana ${ }^{2}$ e Marco Aurélio Cestare ${ }^{2}$
}

\author{
${ }^{1}$ Eng. Agr., Dr., Professor. FEIS/UNESP. Av. Brasil, 56. Caixa Postal 31. Ilha Solteira, SP 15385-000 ftadeu@bio.feis.unesp.br \\ ${ }^{2}$ Graduando de Agronomia. FEIS/UNESP.
}

\begin{abstract}
RESUMO
O experimento foi desenvolvido na safra 1998/99, na Fazenda de Ensino e Pesquisa da UNESP, em Selvíria (MS), em solo com textura argilosa. O objetivo foi avaliar a eficácia de herbicidas aplicados em pré-emergência e avaliar os efeitos tóxicos sobre a cultura do arroz. O cultivar de arroz utilizado foi Caiapó. O delineamento experimental adotado foi o de blocos ao acaso, com dez tratamentos e quatro repetições. Os tratamentos foram os seguintes: trifluralin (1200 g/ha), oxyfluorfen (120 e $240 \mathrm{~g} / \mathrm{ha})$, clomazone (500 g/ha), trifluralin+oxyfluorfen (1800+120 e 1200+240 g/ha), clomazone +oxyfluorfen (500+240 $\mathrm{g} / \mathrm{ha}$ ), pendimethalin (1250 g/ha) e testemunhas capinada e sem capina. Avaliou-se a eficácia dos tratamentos no controle das plantas daninhas e o efeito dos mesmos na produtividade da cultura. Concluiu-se que os herbicidas e doses testados provocam sintomas de toxicidade inicial às plantas de arroz, os quais desaparecem 45 dias após a aplicação e não afetam a produtividade da cultura. As principais espécies daninhas que ocorreram no experimento foram: Ipomoea acuminata, Commelina benghalensis, Blainvillea rhomboidea e Amaranthus deflexus. Os tratamentos oxyfluorfen ( $240 \mathrm{~g} / \mathrm{ha})$ e clomazone+oxyfluorfen $(500+240 \mathrm{~g} / \mathrm{ha})$ proporcionaram os melhores controles das plantas daninhas estudadas. A interferência das plantas daninhas na testemunha no mato reduziu a produtividade da cultura em $56 \%$ em relação à testemunha no limpo.
\end{abstract}

Palavras-chave: Oryza sativa, interferência.

\section{ABSTRACT \\ Efficiency of herbicides in preemergence control of weeds in a rice crop}

The experiment was developed in 1998/99, in the UNESP Experimental Farm, in Selvíria (MS), on soil with a loamy texture. The objective of the present work was to evaluate the tolerance and efficiency of preemergence herbicides on rice and observe the effects of the treatments on the productivity of the crop. The rice strain used was Caiapó. The experimental design was a complete randomized block with ten treatments and four replications. The treatments were the following: trifluralin (1200 g/ha), oxyfluorfen (120 and $240 \mathrm{~g} / \mathrm{ha})$, clomazone $(500 \mathrm{~g} / \mathrm{ha})$, trifluralin + oxyfluorfen $(1800+120 \mathrm{and} 1200+240 \mathrm{~g} /$ ha), clomazone + oxyfluorfen $(500+240 \mathrm{~g} / \mathrm{ha})$, pendimethalin $(1250 \mathrm{~g} / \mathrm{ha})$ and controls with and without weeds. The efficiency of the treatments was evaluated in terms of the control of the weeds and the effect of the treatments on the productivity of the crop. The herbicides and doses tested in the experiment provoked symptoms of initial toxicity to the rice plants, which disappear 45 days after application and these do not affect the productivity of the crop. The main weed species that appeared in the experiment included: Ipomoea acuminata, Commelina benghalensis, Blainvillea rhomboidea and 
Amaranthus deflexus. The treatments oxyfluorfen $(240 \mathrm{~g} / \mathrm{ha})$ and clomazone + oxyfluorfen $(500+240 \mathrm{~g} / \mathrm{ha})$ provided the best control of the studied weed. The interference of the weeds reduced the productivity of the crop in $56 \%$ in relation to the weed free control.

Key words: Oryza sativa, interference.

\section{INTRODUÇÃO}

A cultura da arroz está sujeita a vários tipos de danos, como o ataque de pragas e a ocorrência de doenças e plantas daninhas, que dificultam o seu desenvolvimento e reduzem sua produtividade.

No que diz respeito às plantas daninhas, as perdas mundiais de produção ao ano na cultura do arroz são de $10 \%$, devido à competição com as mesmas. (ANDEF, 1987).

Blanco (1985) cita que as perdas de produção de arroz, no Brasil, devido à ocorrência de plantas daninhas, pode ser de 45 a $95 \%$. Outros autores observaram valores semelhantes, variando um pouco conforme a região. Desta forma Carson (1975), Burga \& Tozani (1980), Fisher et al. (1980), Domingues (1981) e Alcântara et al. (1982), constataram prejuízos de $58,95,51,56$ a 71 e $96 \%$, respectivamente, para a cultura de arroz convivendo durante todo o ciclo com as plantas daninhas.

Existem diferentes métodos de se controlar as plantas daninhas na cultura do arroz. A utilização de herbicidas é uma boa opção devido a eficiência do controle e ao alto rendimento operacional que se consegue nas aplicações.

O objetivo do presente trabalho foi avaliar a eficácia de herbicidas aplicados em pré-emergência e avaliar os efeitos tóxicos sobre a cultura do arroz.

\section{MATERIAL E MÉTODOS}

O experimento foi conduzido na safra 1998/99, com semeadura em 24/12/98, em área irrigada da Fazenda de Ensino e Pesquisa (FEP) da UNESP - FEIS, localizada no município de Selvíria (MS), enquadrada em região de Cerrado, no sudeste do Mato Grosso do Sul. O solo da área experimental é classificado como Latossolo vermelho-escuro, textura argilosa, com $48 \%$ de argila, $7 \%$ de silte e $45 \%$ de areia. O teor de matéria orgânica era de $1,9 \%$ e o pH (em água) de 6,0 .

Antes da montagem do experimento a área foi preparada convencionalmente com uma aração e duas gradagens niveladoras, sendo a última imediatamente antes da semea- dura, de modo a eliminar as plantas daninhas que já haviam germinado. $\mathrm{O}$ cultivar de arroz utilizado foi o Caiapó, semeado manualmente no espaçamento de $0,5 \mathrm{~m}$ entre linhas.

Os tratos culturais realizados na área experimental, foram os normais exigidos pela cultura no que diz respeito às adubações, irrigações e controle de pragas e doenças. A adubação foi realizada concomitantemente à semeadura, utilizando-se a fórmula comercial 8-28-16, à base de $240 \mathrm{~kg} / \mathrm{ha} \mathrm{e}$ a adubação de cobertura, aos 25 dias após a semeadura, com uréia a $100 \mathrm{~kg} / \mathrm{ha}$. Para o controle de pragas, principalmente cupins, foi colocado no sulco de plantio o inseticida Furadan $100 \mathrm{G}$, na dose de $5,0 \mathrm{~kg} / \mathrm{ha}$. Quanto às irrigações, foram realizadas sempre que necessário, procurando-se suprir a necessidade de água da cultura, através de um sistema de irrigação por pivô-central.

O delineamento experimental adotado foi o de blocos ao acaso, com dez tratamentos e quatro repetições. Cada parcela constou de oito linhas da cultura com $4 \mathrm{~m}$ de comprimento e $4 \mathrm{~m}$ de largura, totalizando $16 \mathrm{~m}^{2}$. A área total do experimento (48 parcelas) foi de $768 \mathrm{~m}^{2}$.

As aplicações dos herbicidas foram realizadas em préemergência da cultura e das plantas daninhas, com um pulverizador costal pressurizado $\left(\mathrm{CO}_{2}\right)$ com pressão constante de $3,2 \mathrm{~kg} / \mathrm{cm}^{2}$, provido de tanque com capacidade de dois litros (garrafas descartáveis), e com barra equipada com quatro bicos do tipo leque, marca Teejet 110.03 , espaçados de $0,5 \mathrm{~m}$. O volume de calda aplicado foi de $300 \mathrm{l} / \mathrm{ha}$. Na ocasião o solo encontrava-se úmido, temperatura do ambiente, marcada no início, de $29^{\circ} \mathrm{C}$, umidade relativa do ar de $66 \%$ e velocidade do vento inferior a $6 \mathrm{~km} / \mathrm{h}$.

As avaliações de eficácia dos produtos foram realizadas através de uma escala visual, comparando-se os tratamentos com a testemunha no mato e estimando-se a percentagem de controle ( 0 a $100 \%$ ) obtida pelos herbicidas. Foram considerados eficazes os tratamentos com média de controle igual ou superior a $80 \%$. As avaliações de seletividade também foram realizadas com escala visual de notas, comparando-se os tratamentos com a testemunha no limpo e estimando-se a percentagem de fitotoxicidade ( 0 a $100 \%$ ) causada pelos herbicidas. As avaliações foram realizadas aos $15,30 \mathrm{e}$ 45 dias após a aplicação (DAA) dos herbicidas. Os dados apresentados na Tabela 1 referem-se à avaliação de controle aos 45 DAA, considerada a mais importante para o trabalho.

A avaliação de produtividade foi realizada aos 118 dias após a semeadura coletando-se os grãos da área útil, ou seja, 
Eficiência de herbicidas no controle pré-emergente...

Tabela 1. Percentagem média de controle das plantas daninhas aos 45 dias após a aplicação dos herbicidas. Selvíria, MS. $1998 / 99$.

\begin{tabular}{|c|c|c|c|c|c|}
\hline \multirow{2}{*}{ TRATAMENT $O^{1}$} & \multirow{2}{*}{$\begin{array}{c}\text { DOSE } \\
(\mathrm{g} / \mathrm{ha})\end{array}$} & \multicolumn{4}{|c|}{ CONTROLE AOS 45 DAA (\%) } \\
\hline & & $\begin{array}{c}\text { Ipontoea } \\
\text { acuminata }\end{array}$ & $\begin{array}{l}\text { Commelina } \\
\text { benghalensis }\end{array}$ & $\begin{array}{l}\text { Blainvillea } \\
\text { rhomb oidea }\end{array}$ & $\begin{array}{c}\text { Amaranthos } \\
\text { deflexus }\end{array}$ \\
\hline Testemunha no mato & -- & -- & -- & -- & $-\cdot$ \\
\hline Testemunha no limpo & -- & 100 & 100 & 100 & 100 \\
\hline Trifluralin & 1200 & 64 & 51 & 70 & 94 \\
\hline Oxyflu orfen & 120 & 89 & 80 & 91 & 98 \\
\hline Oxyflu orfen & 240 & 90 & 95 & 96 & 100 \\
\hline Clomazone & 500 & 82 & 95 & 96 & 75 \\
\hline Trifluralin+oxyfluorfen & $1800+120$ & 87 & 80 & 91 & 92 \\
\hline Trifluralin+oxyfluorfen & $1200+240$ & 86 & 96 & 94 & 100 \\
\hline Clomazone+oxyfluorfen & $500+240$ & 95 & 96 & 94 & 100 \\
\hline Pendimethalin & 1250 & 82 & 80 & 80 & 100 \\
\hline
\end{tabular}

${ }^{1}$ Nomes comuns e comerciais: trifluralin $=$ Premerlin $600 \mathrm{CE}$, oxyfluorfen $=$ Galigan $240 \mathrm{CE}$, clomazone $=\mathrm{HBT}-382$, pendimethalin $=$ Herbadox $500 \mathrm{CE}$.

os $4 \mathrm{~m}^{2}$ centrais de cada parcela. As comparações entre as médias de produtividade dos tratamentos foram realizadas pelo teste de Tukey a $5 \%$ de probabilidade.

\section{RESULTADOS E DISCUSSÃO}

O herbicida trifluralin, na dose de $1200 \mathrm{~g} / \mathrm{ha}$, foi eficaz no controle de Amaranthus deflexus, proprocionando um nível médio de controle de 94\%, aos 45 DAA (Tabela 2). Para as espécies Ipomoea acuminata, Blainvillea rhomboidea e Commelina benghalensis, o tratamento foi pouco eficaz. Esses dados estão de acordo com Lorenzi et al. (1994) sobre a eficácia do trifluralin para estas espécies.

O herbicida oxyfluorfen, na dose de $240 \mathrm{~g} / \mathrm{ha}$, foi eficiente no controle de I. acuminata, C. benghalensis, $B$. rhomboidea e $A$. deflexus, proporcionando níveis de controle de $90,95,96$ e 100\%, respectivamente, aos 45 DAA. Na dose de $120 \mathrm{~g} /$ ha também foi eficiente, proporcionando níveis de controle de $89,80,91$ e $98 \%$, respectivamente.

O herbicida clomazone, na dose de $500 \mathrm{~g} / \mathrm{ha}$, foi eficaz no controle de I. acuminata, C. benghalensis e B. rhomboidea, proporcionando níveis médios de controle de 82,95 e $96 \%$, respectivamente, aos 45 DAA. Para $A$. deflexus o tratamento foi considerado pouco eficiente, proporcionando um nivel médio de controle de $75 \%$, aos 45 DAA.

A mistura trifluralin+oxyfluorfen $(1800+120 \mathrm{~g} / \mathrm{ha})$ foi eficaz no controle de $I$. acuminata, $C$. benghalensis, $B$. rhomboidea e $A$. deflexus, proporcionando níveis de controle de $87,80,91$ e $92 \%$, respectivamente, aos 45 DAA. Na dose de $1200+240 \mathrm{~g} /$ ha foi eficaz no controle das mesmas espécies daninhas, proporcionando níveis médios de controle de $87,96,94$ e $100 \%$, respectivamente, aos 45 DAA.

A mistura clomazone +oxyfluorfen $(500+240 \mathrm{~g} / \mathrm{ha})$ foi eficaz no controle de $I$. acuminata, $C$. benghalensis, $B$. rhomboidea e $A$. deflexus, proporcionando níveis de controle de $95,96,94$ e 100\%, respectivamente, aos 45 DAA.

Tabela 2. Infestação (\%) das plantas daninhas na testemunha sem capina. Selvíria, MS. 1998/99.

\begin{tabular}{|c|c|c|c|}
\hline \multirow{2}{*}{ E S P É C IE D A N IN H A } & \multicolumn{3}{|c|}{ IN FE S TA C A A } \\
\hline & $15 \mathrm{DAA}$ & 30 D A A & $45 \mathrm{DAA}$ \\
\hline Ipomoea acuminata (corda-de-viola) & 8 & 10 & 12 \\
\hline Commelina benghalensis (trapoeraba) & 6 & 10 & 12 \\
\hline Blainvillea rhomboidea (erva-palha) & 6 & 8 & 12 \\
\hline Amaranthus deflexus (caruru) & 5 & 7 & 12 \\
\hline TOT AL & 25 & 35 & 48 \\
\hline
\end{tabular}

DAA = dias após a aplicação dos herbicidas 
O herbicida pendimethalin (1250 g/ha) foi eficiente no controle de $I$. acuminata, $C$. benghalensis e $B$. rhomboidea, proporcionando, no entanto, níveis mais baixos de controle, sendo de 82,80 e $80 \%$, respectivamente. Para a espécie $A$. deflexus o controle foi de $100 \%$, aos 45 DAA.

Entre os tratamentos estudados observa-se que 0 herbicida oxyfluorfen $(240 \mathrm{~g} / \mathrm{ha})$ e a mistura clomazone+oxyfluorfen $(500+240 \mathrm{~g} / \mathrm{ha})$ proporcionaram os maiores controles das quatro espécies daninhas.

Todos os herbicidas provocaram sintomas de fitotoxicidade que permaneceram nas plantas até os 30 DAA. Aos 45 DAA não se observou mais nenhum sintoma nas plantas da cultura ( Tabela 3 ).

Os dados de produtividade confirmam os efeitos prejudiciais da competição entre cultura e plantas daninhas, já constatado por outros autores (Carson, 1975; Burga \& Tozani,

Tabela 3. Percentagem média de fitotoxicidade dos herbicidas às plantas de arroz e produtividade da cultura. Selvíria, MS. 1998/99.

\begin{tabular}{|c|c|c|c|c|c|}
\hline \multirow{2}{*}{ T R A T A M E T O } & \multirow{2}{*}{$\begin{array}{l}\text { DOSE } \\
(\mathrm{g} / \mathrm{ha})\end{array}$} & \multicolumn{3}{|c|}{ FIT O T OXICIDA DE $(\%)$} & \multirow{2}{*}{$\begin{array}{c}\text { PRO D T IV ID A D E } \\
(\mathrm{kg} / \mathrm{h} \mathrm{a})\end{array}$} \\
\hline & & $15 \mathrm{DAA}$ & $30 \mathrm{DAA}$ & $45 \mathrm{DAA}$ & \\
\hline Testemunha no mato & -- & -- & -- & -- & 1174 \\
\hline Testemunha no $\operatorname{limpo}$ & $\ldots$ & $\ldots$ & $\ldots$ & - & 2690 \\
\hline T riflu ralin & 1200 & 5,5 & 1,3 & 0,0 & $2350 \mathrm{ab}$ \\
\hline Oxyflu orfen & 120 & 6,0 & 10,0 & 0,0 & 2046 \\
\hline Oxyflu orfen & 240 & 15,3 & 15,8 & 0,0 & 2181 \\
\hline Clom azone & 500 & 7,8 & 7,5 & 0,0 & $1635 \mathrm{ab}$ \\
\hline T rifluralint ox y flu or fen & $1800+120$ & 14,5 & 18,8 & 0,0 & $1837 \mathrm{ab}$ \\
\hline Trifluralint oxyflu orfen & $1200+240$ & 19,0 & 18,8 & 0,0 & 2851 a \\
\hline C lom azonetoxyfluorfen & $500+240$ & 16,5 & 18,8 & 0,0 & $1974 \mathrm{ab}$ \\
\hline Pendimethalin & 1250 & 5,5 & 5,0 & 0,0 & $2024 \mathrm{ab}$ \\
\hline $\mathrm{M}$ édia $\mathrm{G}$ e ral & & & & & 2076,2 \\
\hline Teste F (tratamentos) & & & & & $3,03 * *$ \\
\hline Coeficiente de $\mathrm{V}$ ariação $(\%)$ & & & & & 27 \\
\hline $\operatorname{DM~S}(5 \%)$ & & & & & 1363,2 \\
\hline
\end{tabular}

Obs.: Médias seguidas por letras iguais não diferem entre si pelo teste de Tukey a $5 \%$ de probabilidade.

1980; Fisher et al., 1980; Domingues, 1981; Alcântara et al., 1982 e Blanco, 1985). A convivência com as plantas daninhas prejudicou a produtividade da cultura, principalmente na testemunha no mato.

\section{CONCLUSÕES}

Os herbicidas trifluralin, oxifluorfen, clomazine e pendimethalin provocam inicialmente, aos 15 e 30 DAA, sintomas de toxicidade às plantas de arroz, cultivar Caiapó, os quais não afetam a produtividade da cultura.

O herbicida trifluralin ( $1200 \mathrm{~g} / \mathrm{ha})$ é eficaz no controle de Amaranthus deflexus.

O herbicida oxyfluorfen ( $120 \mathrm{~g} / \mathrm{ha}$ ) é eficaz no controle de I. acuminata, C. benghalensis, B. rhomboidea e A. deflexus. Na dose de $240 \mathrm{~g} /$ ha é mais eficaz no controle de $C$. benghalensis.

O herbicida clomazone ( $500 \mathrm{~g} / \mathrm{ha}$ ) é eficiente no controle de I. acuminata, C. benghalensis e B. rhomboidea.

A mistura trifluralin+oxyfluorfen $(1800+120 \mathrm{~g} / \mathrm{ha})$ é eficaz no controle de I. acuminata, $C$. benghalensis, $B$. rhomboidea e $A$. deflexus. Na dose $1200+240$ g/ha é mais eficaz no controle de $C$. benghalensis.

A mistura clomazone+oxyfluorfen $(500+240 \mathrm{~g} / \mathrm{ha})$ é eficaz no controle de I. acuminata, C. benghalensis, $B$. rhomboidea e $A$. deflexus.

A interferência das plantas daninhas na testemunha no mato reduziu a produtividade da cultura em $56,4 \%$ em relação à testemunha no limpo.

\section{LITERATURA CITADA}

ALCÂNTARA, E.N.; CARVALHO, D.A.; SOUSA, A.F. Épocas críticas de competição das plantas daninhas com o arroz de sequeiro (Oriza sativa L.) In: CONGRESSO DE LA ASSOCIACION LATINOAMERICANA DE MAZELAS, 6, Campinas. Resumos... p. 35-36. 1982.

ASSOCIAÇÃO NACIONAL DE DEFENSIVOS AGRÍCOLAS - ANDEF. Defesa vegetal. São Paulo,SP: ANDEF, 1987. $19 \mathrm{p}$. 
Eficiência de herbicidas no controle pré-emergente...

BLANCO, H.G. Ecologia das plantas daninhas - competição de plantas daninhas em culturas brasileiras. In: Controle Integrado de Plantas Daninhas. $2^{\mathrm{a}}$ ed. São Paulo: CREA, 1985. p. 42-75.

BURGA, C.A.; TOZANI, R. Competição de plantas daninhas com a cultura do arroz de sequeiro (Oryza sativa L.) In: CONGRESSO BRASILEIRO DE HERBICIDAS E ERVAS DANINHAS, 13, Itabuna. 1980. Resumos... p.22-23. 1980.

CARSON, A.G. Weed competition and some promising herbicide combination for its control in upland rice (Oryza sativa L.). Ghana Journal of Agricultural Science, v.8, n.3, p.223-230. 1975.
DOMINGUES, E.P. Efeitos do espaçamento e fertilização nitrogenada em cobertura sobre as relações competitivas entre a cultura do arroz de sequeiro (Oryza sativa L.) e a comunidade infestante. Jaboticabal. FCAVJ/ UNESP, Trabalho de Graduação. 1981. 75p.

FISHER, H.H., SABIO, E.A., PASTORES, R.M. Weed control systems for upland rice in the Philippines. Hort. Science, v.15, n.3, p.413. 1980.

LORENZI, H. et al. Manual de identificação e controle de plantas daninhas: plantio direto e convencional. 4ª Ed., Nova Odessa, SP: Plantarum, 1994. 299 p. 
